\title{
Optimizing the Patient Telemedicine Experience in an Orthopaedic Clinic
}

\author{
Margaret A. Sinkler ${ }^{1}$, Joshua D. Dolan ${ }^{1}$, Michael J. Steflik ${ }^{1}$, Peter Harimtepathip, MD ${ }^{2}$, J. Shell Cox ${ }^{1}$, \\ Stephen A. Parada, MD ${ }^{2}$ \\ 1. Orthopaedics, Augusta University Medical College of Georgia, Augusta, USA 2. Orthopaedic Surgery, Augusta \\ University Medical College of Georgia, Augusta, USA
}

Corresponding author: Joshua D. Dolan, jdolan@augusta.edu

\begin{abstract}
With the rapid advancements in today's technology, the telemedicine model of healthcare has become an increasingly useful tool for healthcare providers and patients to interact outside of the confines of a traditional office visit. As a result of the COVID-19 pandemic, many providers have been forced to adopt a component of telemedicine into their practice. In an effort to improve the telemedicine system for continued use, 519 patients in an orthopaedic clinic at a Level One academic system were surveyed on their willingness and confidence to use telemedicine in future orthopaedic visits. Though most patients reported that they had been unwilling to use telemedicine for their current visit, the majority were neutral or willing to use telemedicine in the future. In this study, we present some challenges to the orthopaedic telemedicine visit, patient sentiment towards the current and future use of telemedicine in orthopaedics, as well as possible direction for improvement so that telemedicine can be better incorporated into the orthopaedic clinic.
\end{abstract}

Review began 07/12/2021 Review ended 07/20/2021 Published 08/04/2021

๑) Copyright 2021

Sinkler et al. This is an open access article distributed under the terms of the Creative Commons Attribution License CC-BY 4.0., which permits unrestricted use, distribution, and reproduction in any medium, provided the original author and source are credited.
Categories: Orthopedics, Ouality Improvement, Healthcare Technology

Keywords: telemedicine in orthopedics, telemedicine experience, orthopedic telemedicine, covid-19 telemedicine, covid-19 orthopedics, orthopedic visits, orthopedics, optimization

\section{Introduction}

The patient experience has been defined as “everything we say and do that affects our patients' thoughts, feelings, and well-being”, and is increasingly being recognized as a fundamental component of value-based care. Clinical studies have demonstrated that improvements to the patient experience yield greater satisfaction, increased adherence to treatment, and a maintained level of clinical outcomes [1-4].

With the advent of the novel coronavirus disease (COVID-19), virtual and off-site patient encounters have drastically increased [5]. In order to curb the spread of the virus, the Center for Disease Control and Prevention (CDC) recommended social distancing guidelines and limitation of physical contact between individuals in close proximity [6]. Telemedicine was first discussed and implemented as a concrete solution to continue medical care in a socially distanced setting during the West Africa Ebola outbreak in 2015 [7]. In a survey conducted of all the enhanced recovery after surgery (ERAS) orthopaedic programs, it was seen that 106 of the 168 respondents utilize telehealth services. Of these, 83\% state that the COVID-19 pandemic was the impetus for the implementation of the service [8].

The telemedicine model protects both patients and clinicians from potential exposure, is conducive to selfquarantine, and is a 21st-century approach to triage patients [9]. The model has been successfully implemented largely through technological improvements, high-speed internet, and access to smartphones [7]. Many patients have had, or will have, their first telemedicine visit with a provider because of COVID-19. Although telemedicine servers have been largely implemented in the wake of the pandemic, there is need for discussing patient preferences and optimizing the system for continued use moving forward.

The purpose of this study is to evaluate patients' current perception and to identify current limitations of telemedicine visits in an orthopaedic clinic. The specific aim of the study is to identify patient preferences related to telemedicine, willingness to participate, and the device that they intend to use. By gathering this information, we can optimize the telemedicine experience moving forward to ensure the best possible patient experience in the future.

\section{Materials And Methods}

Following Institutional Review Board (IRB) approval, informed consent and patient survey forms were distributed by front desk members at an outpatient orthopaedic surgery clinic upon arrival for in-person consultation from $11 / 23 / 2020$ to $1 / 19 / 2021$. If patients agreed to the consent, surveys were completed in full alongside intake forms prior to their orthopaedic consult. The questionnaires were collected by an authorized investigator and stored on a secured cloud drive. The inclusion criteria for the study were males 


\section{Cureus}

and females presenting to the orthopaedic clinic at the institution for either first visit or recheck, who were above the age of 18 years and were capable of consenting to the study. Participation in the study was completely voluntary and had no impact on services rendered during the physical visit in which they were given the survey. Of the 640 surveys that were handed out, 516 were completed and eligible for inclusion in the survey. No direct or indirect patient identifiers were collected, and all 18 characteristics of the Health Insurance Portability and Accountability Act (HIPAA) de-identification standard were met.

The Appendix includes the 11-question survey that was given to patients in the clinic. Responses were analyzed using a Mann-Whitney U Test in cases of non-parametric data or a Chi-square analysis in the case of categorical data. The R 4.0.0 ${ }^{\mathrm{TM}}$ statistical package was used for statistical calculation.

\section{Results}

A total of 519 surveys were obtained from outpatient orthopaedic clinics between 11/23/2020 to 1/19/2021 with an $80.6 \%$ survey completion rate. A summary of respondent demographics may be found in Table 1 . The full survey questionnaire is provided in the Appendix.

\begin{tabular}{|l|l|l|}
\hline Question & Answer & $n(\%)$ \\
\hline Sex & Male & 203 (40.03) \\
\hline & Female & $304(59.96)$ \\
\hline & Less than high school & $22(4.26)$ \\
\hline Education & High school & $113(21.90)$ \\
& Some college & $147(28.49)$ \\
\hline & College & $110(21.32)$ \\
\hline Median Age & Graduate degree or above & 112 (21.71) \\
\hline
\end{tabular}

TABLE 1: Demographic information of survey participants

Over three-fourth (86.5\%) of the orthopaedic patients surveyed were neutral or opposed to current telemedicine visits while only $13.5 \%$ (69) surveyed patients had a preference or strong preference for telemedicine visits. Approximately $33.1 \%$ of patients had a strong in-person preference and $8.0 \%$ had a strong preference for telemedicine (Figure 1). However, 78.2\% (401) of patients possessed a neutral or willing outlook on future telemedicine visits compared to $21.8 \%$ (112) that were either unwilling or very unwilling to participate in future telemedicine visits (Figure 1). 


\section{Cureus}
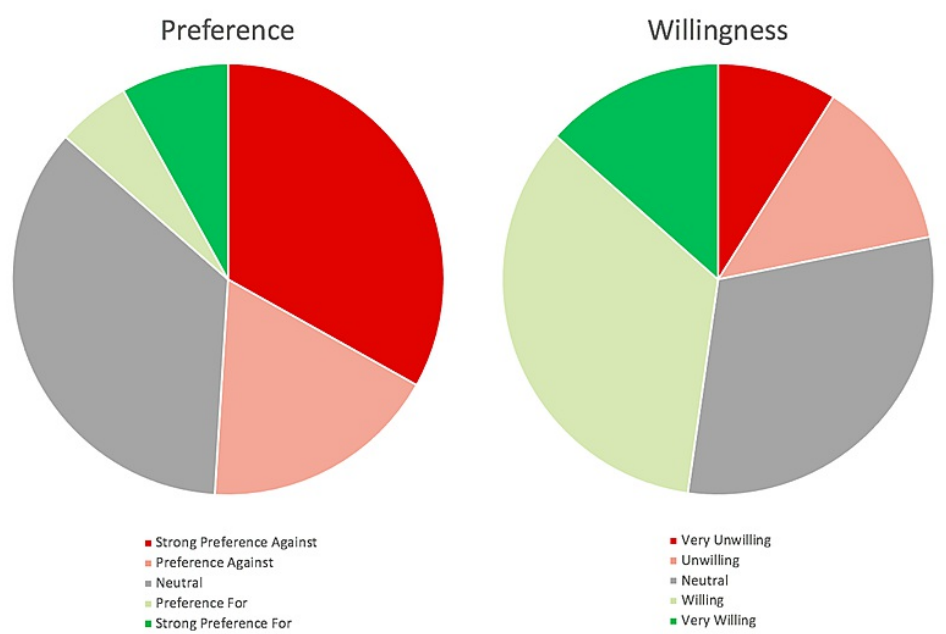

FIGURE 1: Percentage breakdown of surveyed patients demonstrating their preference and willingness to participate in telemedicine visits.

After recording patient responses to the survey given prior to their appointment, their preference for a telemedicine visit was compared to their willingness to participate in a future telemedicine appointment. The chart on the left illustrates current patient sentiment regarding their preference for a telemedicine appointment versus an in-person appointment at the time of the survey. The results point to a majority preferring an in-person visit versus telemedicine. The chart on the right shows patient willingness to use telemedicine in the future and shows a dramatic increase in the number of patients willing to use telemedicine moving forward.

Patient comfort level using electronic devices was directly related to their willingness for future telemedicine visits as well as the likelihood they preferred virtual visits currently $(\mathrm{p}<0.05)$ shown in Figure 2. Exceptions to this include those who reported slight discomfort versus neutral comfort in using their electronic devices $(\mathrm{p}=0.50)$ and those who reported slightly comfortable versus those who reported extreme comfort using their electronic devices $(\mathrm{p}=0.68$ ). Additionally, those less comfortable with their electronic devices expressed a strong preference for in-person visits while those who reported being more comfortable with their devices expressed a neutral preference for in-person versus telemedicine visits. This trend is significant except for when comparing those who reported neutral comfort with their devices with those who reported slight discomfort, slight comfort, and extreme comfort with their devices $(\mathrm{p}=0.09, \mathrm{p}=0.48$, $\mathrm{p}=0.70$, respectively). There was also no statistical difference in current preference between those who expressed slight and extreme comfort $(\mathrm{p}=0.67)$. 


\section{Cureus}

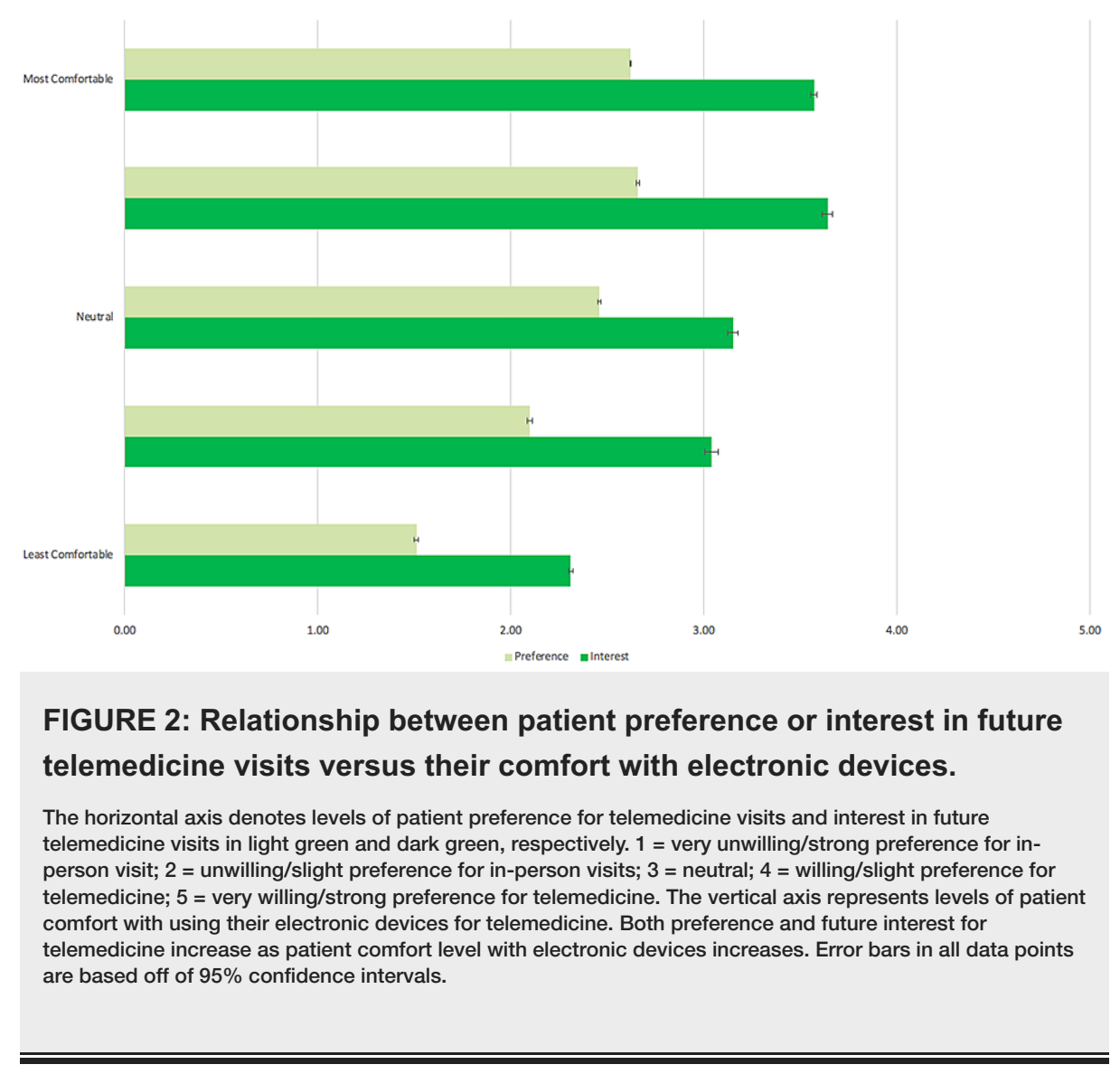

The majority of patients have had no direct or familial experience with prior telemedicine visits (74\%). Those with prior experiences with telemedicine expressed greater willingness to participate in future telemedicine visits versus those with no history $\left(\mathrm{p}=3.97 \times 10^{\wedge}-5\right)$. Those with a history of telemedicine visits had more neutral preferences for in-person versus telemedicine visits as opposed to those with no history of telemedicine, who had a stronger in-person visit preference $\left(\mathrm{p}=8.00 \times 10^{\wedge}-5\right)$.

The majority of patients reported that they would use a cell phone as their primary device (63.75\%) followed by computer (15.41\%), laptop (13.29\%), and tablet (12.89\%). A majority of respondents reported a second available device (65.85\%) (Table 2). Those without stable or reliable internet were found to have a more neutral opinion on current visit type compared to those with $\mathrm{Wi}-\mathrm{Fi}$, who expressed greater preference for telemedicine $(\mathrm{p}<0.05)$. There was also a significant difference when comparing those without reliable Wi-Fi, who were less interested in the future of telemedicine visits, compared to those with access to reliable Wi-Fi, who were more neutral $(\mathrm{p}<0.05)$. 


\section{Cureus}

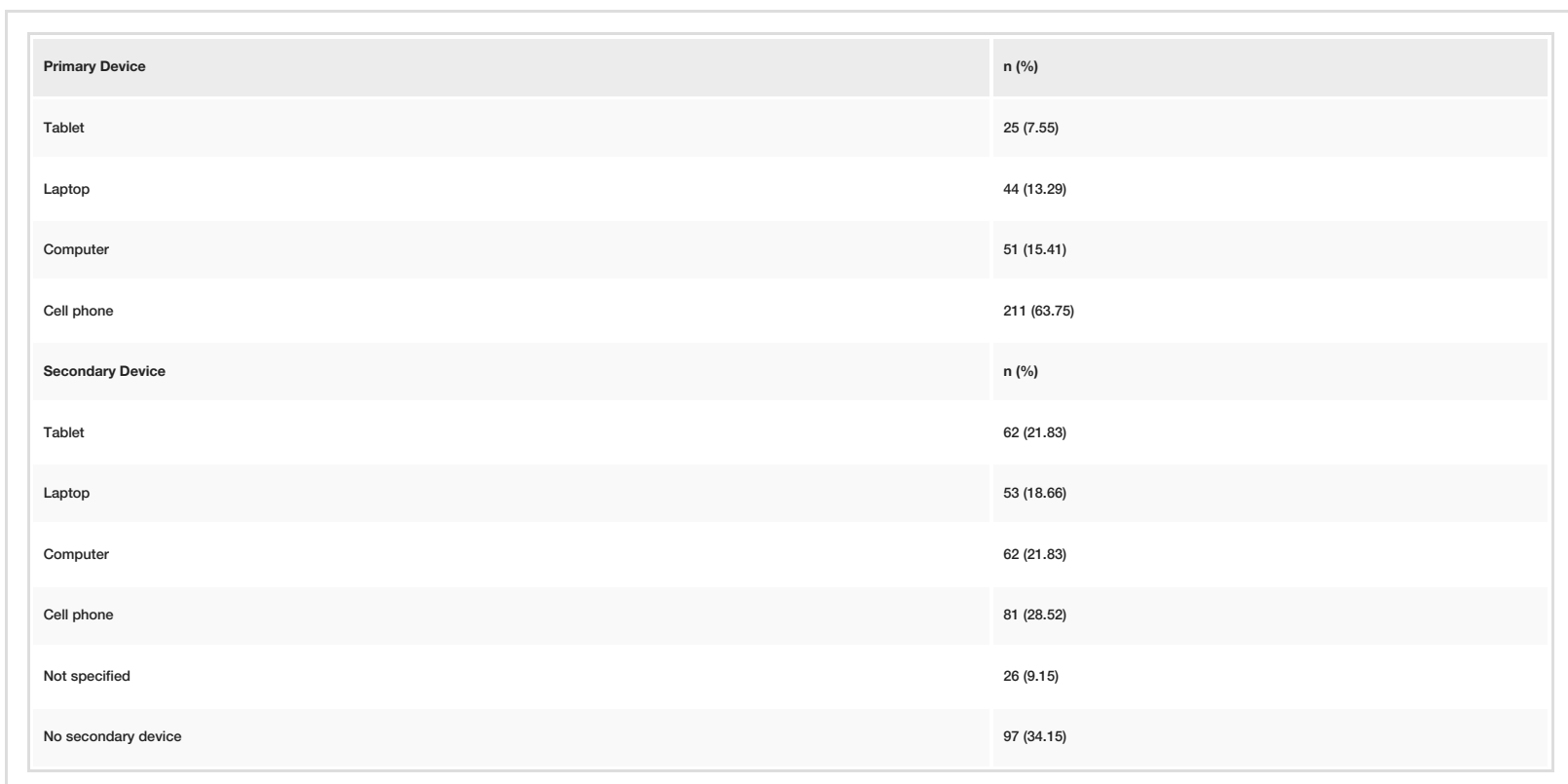

TABLE 2: Device used for telemedicine visits by survey respondents

\section{Discussion}

The novel coronavirus disease (COVID-19) pandemic had an immediate impact on in-person evaluation of patients, which has been demonstrated in multiple studies across the United States (US). A study conducted in an outpatient orthopaedic spine clinic in Utah during the initial four weeks of the COVID-19 pandemic, starting from March 16, 2020, showed that overall clinic visits decreased from 417 to 322 and new patient visits decreased from $28 \%$ to $20 \%$. Of the 322 visits, 318 (98.7\%) were performed via telemedicine [10]. In a survey given to the 3,400 members of the American Association of Hip and Knee Surgeons (AAHKS), starting March 20, 2020, over $90 \%$ of the surgeons surveyed reported a reduction in patient load in the first three months. Over time, the reported reduction in clinic volume dropped to $73 \%$ by mid-June, 2020 , and $59 \%$ by September 2020. By September 2020, 35\% of respondents were using telemedicine [11]. Therefore, the adoption and incorporation of telemedicine into practice had a large impact on maintaining clinic volumes during the COVID-19 pandemic.

Telemedicine is not a new concept in medicine as initial manuscripts appeared in the late 19th century. Even prior to COVID-19, improvements in technology and devices had increased the relevance of telemedicine visits. Interest in telemedicine was evident earlier too, as the Kaiser Family Foundation found increasing rates of employee telemedicine coverage in 2019 [12]. While there are known benefits to telemedicine visits including increased access, lower cost, and high patient satisfaction rates, several barriers exist preventing the widespread use of telemedicine visits including implementation cost, lack of awareness, inefficiencies, medicolegal liability implications, and difficulty in examining patients virtually [13].

Our study was performed at a Level One academic center in the Southeast. In a recent study using 175 ERAS participating orthopaedic programs, there was a nationwide increase in the use of telemedicine with $74.4 \%$ of programs in the Northeast, $69.6 \%$ of programs in the West, $66.1 \%$ in the South, and $45.7 \%$ in the Midwest [8]. The largest changes in telemedicine visits were correlated to COVID-19 hot spots. New York state saw a $550 \%$ increase, California a 900\% increase, and Texas a 1200\% increase [8]. Therefore, our study most accurately reflects the current patient perception of telemedicine visits in the Southeast region.

Within our study, a majority of patients reported feeling neutral to opposed with regard to telemedicine visits. The J.D. Power survey in 2019 showed that $29 \%$ of patients did not believe that telemedicine services were available to them and $37 \%$ did not know if their provider offered such services [14]. Patients who had a telemedicine visit in the past were more likely to be willing to participate in future telemedicine visits. This finding agrees with prior studies that have compared telemedicine studies to in-person visits in orthopaedic clinics in multiple regions of the US [15-19].

The majority of participants in our study identified that they would utilize a cell phone as their main device and their comfort with the device directly correlated to their willingness to partake in telemedicine visits. This can be used to optimize the virtual visit towards our patients based on the selected software. Additionally, high-quality video consultations require 4-10 Mbps of Wi-Fi [15]. With the rapid advancements in technology and internet availability today, this requirement becomes less and less of an 
issue.

Prior to COVID-19, a 2013 study at UC Irvine evaluated telemedicine use after total joint arthroplasty where 34 patients had a telemedicine follow-up and 44 patients had an in-person follow-up. Post-operative telemedicine visits yielded higher satisfaction rates [16]. Additionally, in 2015, a prospective randomized control trial at Vanderbilt using 24 patients in an orthopaedic trauma center showed no significant difference in patient satisfaction between telemedicine and in-person visits [17]. In 2019, a study of 167 patients at a pediatric fracture clinic in Pennsylvania found similar satisfaction levels between groups that received telemedicine or in-person care [18]. During the COVID-19 pandemic, a prospective randomized control study in Philadelphia in 2020 had 66 patients utilizing telemedicine for post-operative visits after rotator cuff repair. Patients demonstrated similar pain scores and overall higher satisfaction scores using telemedicine when compared to in-person visits [19]. These studies show that long term implementation of telemedicine visits in orthopaedic clinics is a reasonable and feasible option.

Future studies should shift towards implementing and validating a universal telemedicine model in orthopaedic clinics and evaluating the longevity of telemedicine once the COVID-19 pandemic resolves. Provider feedback and perceived telemedicine limitations will be another critical direction that should be incorporated towards the improvement of the platform. In 2020, the Cleveland Clinic Foundation proposed a universally applicable model using a centralized appointment desk and a virtual short questionnaire that assesses severity. Patients were then stratified into groups according to their problem with high-risk patients directed either to the ED or scheduled for a routine visit [20]. Using this telemedicine triage model, the ease and accessibility of telemedicine can be applied and paired with the necessary in-person appointments, as requested and optimized through physician feedback, to improve the quality of orthopaedic healthcare and improve time efficiency for both patient and provider.

Limitations to the study include a single point of survey, that is the surveys were gathered at one Level One academic training site, and therefore the results may vary when compared to other centers. The surveys were gathered during one time period, that is during the pandemic, and may not reflect opinions in telemedicine following the pandemic. The respondents were not required to complete all survey questions. The study did not include specific aspects of medical care received by the participants to better classify who would benefit from telemedicine visits.

\section{Conclusions}

In this study, we surveyed the patients of an orthopaedic clinic at a Level One academic training site to identify their preferences for current and future orthopaedic telemedicine appointments. Following the initial impact of COVID-19, many providers have incorporated an aspect of telemedicine into their practice, and it continues to remain a popular fixture even as the country opens up. Though more than half of the patients surveyed said they preferred an in-person appointment at the time of their in-person visit, almost $80 \%$ reported they were also either neutral or willing to use telemedicine as a supplemental form of orthopaedic appointment in the future. Our data show that patient confidence in handling their electronic device is a significant factor in both their preference and willingness to use telemedicine. The results of this study reflect a growing trend in the use of technology in medicine as a way to increase the efficiency and efficacy of care. Even though this study involved only one site in the Southeast and a narrow period of time and sentiment, similar results have been found in other major populations at different times. Moving forward, studies that incorporate these existing data should consider investigating the longevity of a streamlined, universal telemedicine platform for use in orthopaedics.

\section{Appendices}


Telehealth Visits in an Orthopaedic Clinic

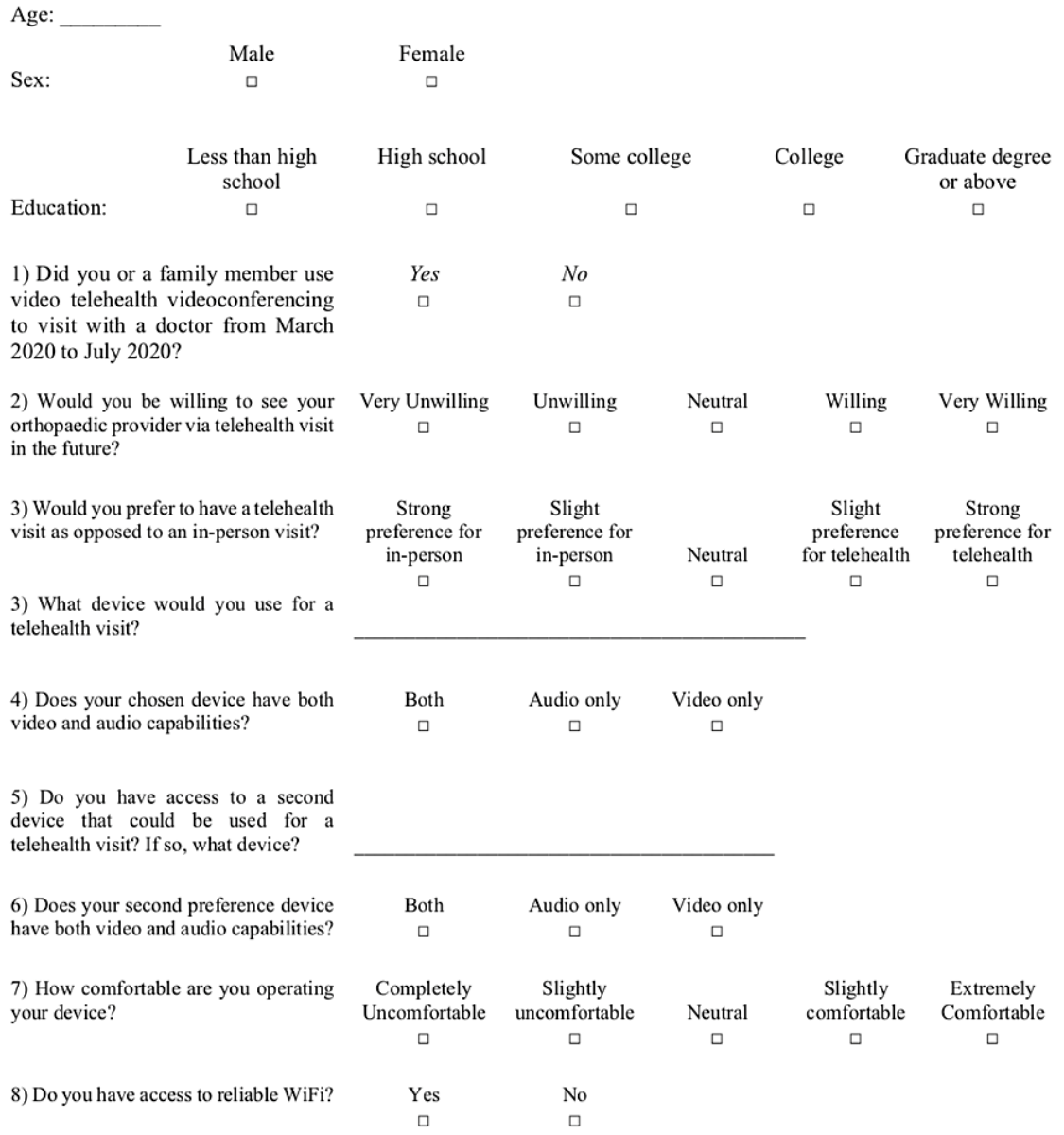

FIGURE 3: Telemedicine survey given to patients at the time of their visit.

\section{Additional Information \\ Disclosures}

Human subjects: Consent was obtained or waived by all participants in this study. Institutional Review Board, Augusta University issued approval Exempt. DATE: November 17, 2020 TO: Stephen Parada, MD FROM: Augusta University (AU) Committee A PROJECT TITLE: [1632870-2] Optimizing the patient telemedicine experience in an orthopaedic clinic SUBMISSION TYPE: New Project (Response/Follow-Up) ACTION: DETERMINATION OF EXEMPT STATUS DECISION DATE: November 16, 2020 REVIEW CATEGORY: Exemption category \# 2 2- Research that only includes interactions involving educational tests (cognitive, diagnostic, aptitude, achievement), survey procedures, interview procedures, or observation of public behavior (including visual or auditory recording) because the following criteria is met: Any disclosure of the human subjects' responses outside the research would not reasonably place the subjects at risk of criminal or civil liability or be damaging to the subjects' financial standing, employability, educational advancement, or reputation; Thank you for your submission of Response/Follow-Up materials for this New Project. The Augusta University (AU) Committee A has determined this project is EXEMPT FROM IRB REVIEW according to federal regulations, 45 CFR 46 (DHHS) 2018 Requirements. Animal subjects: All authors have confirmed that this study did not involve animal subjects or tissue. Conflicts of interest: In compliance with the ICMJE uniform disclosure form, all authors declare the following: Payment/services info: All authors have declared that no financial support was received from any organization for the submitted work. Financial relationships: All authors have declared that they have no financial relationships at present or within the previous three years with any organizations that might have an interest in the submitted work. Other relationships: All authors have declared that there are no other relationships or activities that could appear to have influenced the submitted work.

\section{References}


1. Graham B, Endacott R, Smith JE, Latour JM: 'They do not care how much you know until they know how much you care': a qualitative meta-synthesis of patient experience in the emergency department. Emerg Med J. 2019, 36:355-63. 10.1136/emermed-2018-208156

2. Kneeland PP, Burden M: Annals for hospitalists inpatient notes - patient experience as a health care value domain in hospitals. Ann Intern Med. 2018, 168:HO2-3. 10.7326/M18-0231

3. Defining patient experience: 'everything we say and do' . (2016). Accessed: May 5, 2020: https://www.thehospitalist.org/hospitalist/article/121917/defining-patient-experience-everything-we-say-and-do.

4. Stein SM, Shah SS, Carcich A, McGill M, Gammal I, Langino M, Mauri T: A novel approach to improving patient experience in orthopedics. Am J Med Qual. 2017, 32:655-60. 10.1177/1062860617691124

5. World Health Organization declares COVID-19 a 'pandemic'. Here's what that means . (2020). Accessed: May 5, 2020: https://time.com/5791661/who-coronavirus-pandemic-declaration/.

6. COVID-19: frequently asked questions. (2020). Accessed: February 19, 2020: https://www.cdc.gov/coronavirus/2019-ncov/faq.html.

7. Ohannessian R, Duong TA, Odone A: Global telemedicine implementation and integration within health systems to fight the COVID-19 pandemic: a call to action. JMIR Public Health Surveill. 2020, 6:e18810. $10.2196 / 18810$

8. Parisien RL, Shin M, Constant M, Saltzman BM, Li X, Levine WN, Trofa DP: Telehealth utilization in response to the novel coronavirus (COVID-19) pandemic in orthopaedic surgery. J Am Acad Orthop Surg. 2020, 28:e487-92. 10.5435/JAAOS-D-20-00339

9. Hollander JE, Carr BG: Virtually perfect? Telemedicine for COVID-19. N Engl J Med. 2020, 382:1679-81. 10.1056/NEJMp2003539

10. DeKeyser GJ, Brodke DS, Saltzman CL, Lawrence BD: Response to the coronavirus disease 2019 pandemic by the spine division at a level-I academic referral center. J Am Acad Orthop Surg. 2020, 28:1003-8. 10.5435/JAAOS-D-20-00493

11. Stambough JB, Deen JT, Walton SL, Kerr JM, Zarski MJ, Yates AJ Jr, Andrawis JP: Arthroplasty during COVID-19: surveillance of AAHKS members in the first year of the pandemic. Arthroplast Today. 2021, 7:209-15. 10.1016/j.artd.2020.12.029

12. KKF: 2019 employer health benefits survey. (2019). Accessed: February 18, 2020: https://www.kff.org/report-section/ehbs-2019-section-14-employer-practices-and-health-plan-networks/.

13. Makhni MC, Riew GJ, Sumathipala MG: Telemedicine in orthopaedic surgery: challenges and opportunities . I Bone Jt Surg. 2020, 101:1109-15. 10.2106/JBJS.20.00452

14. Telehealth: best consumer healthcare experience you've never tried, says J.D. Power study . (2019). Accessed: February 18, 2020: https://www.businesswire.com/news/home/20191028005168/en/Telehealth-BestConsumer-Healthcare-Experience-You\%E2\%80\%99v....

15. HealthIT.gov: what is the recommended bandwidth for different types of health care providers? . (2019). Accessed: March 3, 2020: https://www.healthit.gov/faq/what-recommended-bandwidth-different-typeshealth-care-providers.

16. Sharareh B, Schwarzkopf R: Effectiveness of telemedical applications in postoperative follow-up after total joint arthroplasty. J Arthroplasty. 2013, 29:918-922.e1. 10.1016/j.arth.2013.09.019

17. Sathiyakumar V, Apfeld JC, Obremskey WT, Thakore RV, Sethi MK: Prospective randomized controlled trial using telemedicine for follow-ups in an orthopedic trauma population: a pilot study. J Orthop Trauma. 2015, 29:e139-45. 10.1097/BOT.0000000000000189

18. Sinha N, Cornell M, Wheatley B, Munley N, Seeley M: Looking through a different lens: patient satisfaction with telemedicine in delivering pediatric fracture care. J Am Acad Orthop Surg Glob Res Rev. 2019, 3:e100. 10.5435/JAAOSGlobal-D-19-00100

19. Kane LT, Thakar O, Jamgochian G, Lazarus MD, Abboud JA, Namdari S, Horneff JG: The role of telehealth as a platform for postoperative visits following rotator cuff repair: a prospective, randomized controlled trial. J Shoulder Elbow Surg. 2020, 29:775-83. 10.1016/j.jse.2019.12.004

20. King D, Emara AK, Ng MK, et al.: Transformation from a traditional model to a virtual model of care in orthopaedic surgery: COVID-19 experience and beyond. Bone Jt Open. 2020, 1:272-80. 10.1302/20463758.16.BJO-2020-0063.R1 